\title{
Rhizospheric life of Salmonella requires flagella-driven motility and EPS-mediated attachment to organic matter and enables cross-kingdom invasion
}

\author{
Kapudeep Karmakar ${ }^{1}$, Abhilash Vijay Nair ${ }^{1}$, Giridhar Chandrasekharan ${ }^{1,4, \dagger}$, \\ Preeti Garai ${ }^{1}$, Utpal Nath ${ }^{1}$, Karaba N. Nataraj ${ }^{2}$, Prakash N.B ${ }^{3}$ and \\ Dipshikha Chakravortty ${ }^{1, *}$
}

\footnotetext{
${ }^{1}$ Department of Microbiology and Cell Biology, Indian Institute of Science, Bangalore, India, ${ }^{2}$ Department of Crop Physiology, University of Agricultural Science, Bangalore, India, ${ }^{3}$ Department of Soil Science and Agricultural Chemistry, University of Agricultural Science, Bangalore, India and ${ }^{4}$ Department of Microbiology, St. Joseph's College Autonomous, Bangalore, India

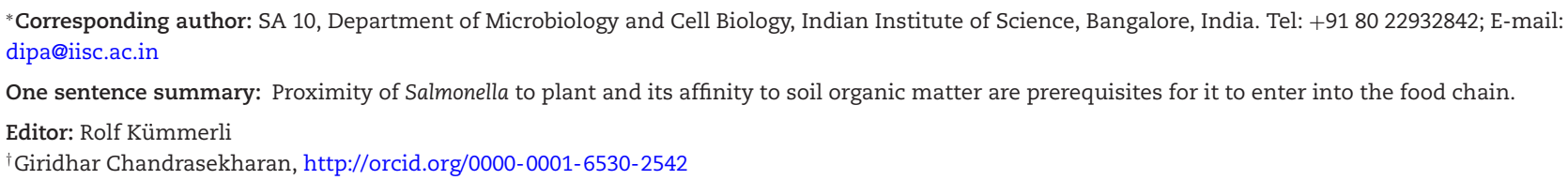

\begin{abstract}
Salmonella is an established pathogen of the members of the kingdom Animalia. Reports indicate that the association of Salmonella with fresh, edible plant products occurs at the pre-harvest state, i.e. in the field. In this study, we follow the interaction of Salmonella Typhimurium with the model plant Arabidopsis thaliana to understand the process of migration in soil. Plant factors like root exudates serve as chemo-attractants. Our ex situ experiments allowed us to track Salmonella from its free-living state to the endophytic state. We found that genes encoding two-component systems and proteins producing extracellular polymeric substances are essential for Salmonella to adhere to the soil and roots. To understand the trans-kingdom flow of Salmonella, we fed the contaminated plants to mice and observed that it invades and colonizes liver and spleen. To complete the disease cycle, we re-established the infection in plant by mixing the potting mixture with the fecal matter collected from the diseased animals. Our experiments revealed a cross-kingdom invasion by the pathogen via passage through a murine intermediate, a mechanism for its persistence in the soil and invasion in a non-canonical host. These results form a basis to break the life-cycle of Salmonella before it reaches its animal host and thus reduce Salmonella contamination of food products.
\end{abstract}

Keywords: Salmonella Typhimurium; flagella; organic matter; EPS; soil; cross-kingdom

Received: 25 January 2019; Accepted: 3 July 2019

CC FEMS 2019. All rights reserved. For permissions, please e-mail: journals.permissions@oup.com 


\section{INTRODUCTION}

The members of the genus Salmonella are known to cause infection through the feco-oral route, primarily due to the consumption of contaminated food (Brandl 2006; Holden, Jackson and Schikora 2015; Wiedemann et al. 2015; Cox et al. 2018). Contaminated food products based on meat, milk, egg and seafood accounted for $67 \%$ of the global Salmonella outbreaks between 2005 and 2011, while contaminated vegetables and fruits accounted for the rest (Nsoesie, Kluberg and Brownstein 2014). Salmonella enterica serovar Typhimurium (S. Typhimurium) is a non-typhoidal serovar that has been well-studied for its gastrointestinal pathogenicity in animals (Jantsch, Chikkaballi and Hensel 2011). However, several studies have reported outbreaks of salmonellosis through consumption of contaminated raw produce, and the possibility of this pathogen being a transkingdom colonist (given its ability to adhere to, invade and proliferate on plants) is being keenly investigated (Van Beneden et al. 1999; Ward et al. 2002; Sivapalasingam et al. 2003; Greene et al. 2008; Mohle-Boetani et al. 2009; Schikora, Garcia and Hirt 2012; Hernandez-Reyes and Adam 2013). Moreover, it has been observed that plants raised from seeds sown in Salmonellacontaminated soil become colonized with the bacteria (Barak and Liang 2008; Barak, Liang and Narm 2008; Barak, Kramer and Hao 2011).

$\mathrm{Gu}$ et al. examined the anatomy of tomato leaves and reported the presence of S. enterica in the vasculature. Salmonella Typhimurium strain MAE110 is capable of entering into the fruits without causing any visible symptoms (Gu et al. 2011). De novo bacterial factors like O-antigen, siderophore and exopolysaccharides are essential for colonization of the plants (Barak et al. 2009; Hao et al. 2012; Cowles et al. 2015). In addition to these pathogen-associated factors, a detailed understanding of the factors governing the attachment of Salmonella to the soil particles can be useful in designing strategies to limit Salmonella colonization. Soil is a heterogeneous mixture of various inorganic and organic components (Pansu and Gautheyrou 2007) and can be divided into bulk soil and the rhizosphere. The preference of Salmonella for these components may vary, which can, in turn, determine its abundance in soil. The distribution of microbes in crop fields can vary spatially and temporally (Smalla et al. 2001). For instance, bacteria can live as epiphytes on the root surface (rhizoplane) or as endophytes inside the plant root tissue (Bodenhausen, Horton and Bergelson 2013). However, there are no reports on a systematic study of proximity-based Salmonella colonization throughout bulk soil, its enrichment in the rhizosphere, its attachment on the rhizoplane and its invasion inside the root tissue. Here, we have attempted to understand the factors governing the attachment of Salmonella to the soil particles and its migration towards the rhizosphere.

We have made use of an artificial potting mixture constituted by vermiculite, perlite and peat moss, which is commonly used for growing plants (Hu et al. 2016; Cabello et al. 2017; Hua et al. 2018), for estimating the preferred attachment site. To test the hypothesis of Salmonella being a cross-kingdom colonist, we examined the pathogenesis in a murine model by feeding the animals with the filtrate from contaminated plant organ. Finally, to complete the disease cycle, we re-established the infection in fresh plants via the murine fecal pellet as the source of infection. Thus, we have addressed the trans-kingdom invasion of Salmonella by following from the free-living stage in the soil to the endophytic stage and then re-establishing the endophytic stage through the soil via passage through the feco-oral route of mice.

\section{MATERIALS AND METHODS}

\section{Bacterial strains, media and culture conditions}

Bacteria were plated either on Luria-Bertani (LB) medium or LB supplemented with kanamycin $(50 \mu \mathrm{g} / \mathrm{mL})$ or chloramphenicol $(50 \mu \mathrm{g} / \mathrm{mL})$ for Salmonella wild type (WT) or mutants $\Delta \operatorname{csgD}$ (Srinandan et al. 2015), $\Delta b c s A$ (Srinandan et al. 2015) and $\Delta$ flic (Garai et al. 2015) from glycerol stocks. $\Delta e n v Z / o m p R$ was provided by Dr Michel Hensel (Division Microbiology, University Osnabrück, Germany). Plating onto Salmonella-Shigella agar (SS agar), a semi-selective differential medium was used to determine S. Typhimurium and Escherichia coli DH5 $\alpha$ populations. Ralstonia solanacearum strain F1C1 was grown on LB agar. Bacterial cultures were grown at $30^{\circ} \mathrm{C}$ (R. solanacearum) for $36 \mathrm{~h}$ or at $37^{\circ} \mathrm{C}$ (S. Typhimurium and E. coli) for $24 \mathrm{~h}$ in respective plates.

\section{Growth conditions for plant}

Arabidopsis thaliana ecotype Col-0 seeds were surface-sterilized and grown on Murashige and Skoog agar media (Bhardwaj et al. 2011). The plates were incubated in a growth chamber (Geotech, Korea). Seedlings were transferred to the autoclaved potting mixture (composed of perlite, vermiculite and peat moss manure in the ratio of 1:1:1). The pots were kept in a growth room at $26^{\circ} \mathrm{C}$. A $16 \mathrm{~h}$ light/ $8 \mathrm{~h}$ dark cycle was used as photoperiod regime with a relative humidity of $75 \%$ and light intensity of 6000 lux. Pots were irrigated with $50 \mathrm{~mL}$ sterile water every third day using a micropipette, taking care to avoid splashing of the component of potting mixture and mixing of bacteria.

\section{Generation of $\Delta$ flic $\Delta$ fljB strain}

The fliC fljB double-deletion mutant was constructed using onestep deletion strategy (Datsenko and Wanner 2000). The $\Delta$ flic bacteria, transformed with a 'lambda red recombinase' expressing plasmid (pKD46) under arabinose-inducible promoter, were grown in LB with ampicillin $(50 \mu \mathrm{g} / \mathrm{mL})$ and kanamycin $(50$ $\mu \mathrm{g} / \mathrm{mL}$ ) and were induced with $10 \mathrm{mM} \mathrm{L}$-arabinose (Sigma) at $30^{\circ} \mathrm{C}$ to an optical denity at $600 \mathrm{~nm}\left(\mathrm{OD}_{600}\right)$ of 0.4 . Electrocompetent bacteria were prepared by washing three times with ice-cold MilliQ water and 10\% glycerol. Polymerase chain reaction (PCR)-amplified product containing the chloramphenicol resistance cassette (from pKD3 plasmid) flanked by sequences upstream and downstream of the genes were obtained (forward: 5'gaggattgctttatcaaaaaccttccaaaagatgaatatcctccttag3' and reverse: $\quad 5^{\prime}$ cacggggctgaataaaacgaaataaattaacgctggagctgcttc $\left.3^{\prime}\right)$. Electroporation was done according to the manufacturer's instructions (Bio-Rad), using 500 ng of PCR product. Transformants were selected on LB agar containing kanamycin (50 $\mu \mathrm{g} / \mathrm{mL})$ and chloramphenicol $(20 \mu \mathrm{g} / \mathrm{mL})$ and were further confirmed by PCR using confirmatory primers designed against flanking loci of the genes.

Enumeration of bacterial population ex situ in bulk soil, rhizosphere, rhizoplane and root tissue

Bacterial strains S. Typhimurium (WT and mutants $\Delta$ envZ/ompR, $\Delta c s g \mathrm{D}$ and $\Delta b c s \mathrm{~A}), \mathrm{E}$. coli DH5 $\alpha$ and R. solanacearum were grown to an $\mathrm{OD}_{600}$ of $0.3,0.3$ and 0.4 , respectively, which corresponds to $\sim 10^{8}$ colony-forming units (CFU)/mL. Cells were centrifuged, re-suspended in phosphate-buffered saline (PBS), diluted $10000-$ fold and mixed thoroughly with the potting mixture $1 \mathrm{~mL}$ suspension/g potting mixture). The final bacterial burden was $10^{4}$ 
CFU/g potting mixture. The mixture was allowed to dry and filled in pots. Ten-day-old A. thaliana Col-0 seedlings were transplanted and CFUs from soil and plant were analyzed 20 days post-inoculation (DPI). CFUs from the non-rhizosphere were collected from the edges of the pot (fresh weight was measured). For the rhizosphere soil sample, the plant was uprooted, and soil adhering to the root was serially diluted and plated for Salmonella colony. The plant roots were weighed (fresh weight) before analyzing rhizoplane and root tissue population. After removal of the soil components from the roots, they were put in sterile PBS and vortexed for $20 \mathrm{~min}$ to remove the surface-adhered rhizoplanic population. The roots were immediately taken out of the suspension and surface-sterilized with $0.5 \%$ bleach treated for 1 min, washed and crushed using mini bead-beader using $1 \mathrm{~mm}$ glass beads (Sigma). The rhizoplanic suspension and tissue suspension were serially diluted and plated. The CFUs for rhizoplane and tissue were normalized to the weight of the roots. A schematic of the experiment is shown in supplementary Fig. S1, available online. The CFUs were plotted against each soil region and one-way analysis of variance (ANOVA) was used to analyze the results.

\section{In vitro adhesion assay}

An artificial potting mixture constituted by vermiculite, perlite and peat moss was used for estimating the preferred attachment site. This mixture has both inorganic (vermiculite, perlite) and an organic component (peat moss), and therefore supports the proper growth of plants. Vermiculite and perlite are silicate clay minerals (Lv, Liu and Rao 2017), which correspond to the inorganic fraction of soil. In vitro soil adhesion was carried out in 24-well microtitre plates. Approximately $10 \mathrm{mg}$ of perlite, vermiculite and organic manure separately was placed in a 24-well plate and saturated with $500 \mu \mathrm{L}$ Salmonella WT or $\Delta$ envZ/ompR, $\triangle \operatorname{csgD}$ and $\triangle b c s A\left(10^{8} \mathrm{CFU} / \mathrm{mL}\right)$ for $1 \mathrm{~h}$. These were then air-dried for $15 \mathrm{~min}$ and incubated at $26^{\circ} \mathrm{C}$ either for $1 \mathrm{~h}$ or $24 \mathrm{~h}$. These were washed with sterile PBS. The particles were either fixed and observed under an electron microscope or dissolved in $10 \mathrm{~mL}$ $\mathrm{PBS}$, and CFU/g of soil was calculated. In another set, the components were treated with Salmonella expressing green fluorescent protein (GFP), and were observed under a confocal microscope. In a different set, perlite, vermiculite and peat moss were mixed in varying amounts with tomato field soil and incubated with WT bacteria. Twenty-four hours post-incubation, Salmonella CFU was plotted.

\section{Ex situ adhesion assay}

Approximately $10^{8}$ cells of WT or mutant strains ( $\triangle$ envZ/ompR, $\Delta c s g \mathrm{D}$ and $\Delta b \operatorname{cs} \mathrm{A}$ ) were mixed per gram of potting mixture. Two hundred milliliters of sterile water was poured in to saturate this artificial soil column after 1, 24 and $48 \mathrm{~h}$. Bacterial CFUs were enumerated in the top $3 \mathrm{~cm}$ layer and the flow-through that comes out of the pot.

\section{Ex situ motility assay}

Pots were radially divided into two concentric regions with a barrier made of filter paper (Merck, Sigma, USA) with pore size 6 $\mu \mathrm{m}$ or $0.2 \mu \mathrm{m}$, or polythene sheet (supplementary Fig. S2a and $\mathrm{b}$, available online). The pore size of $6 \mu \mathrm{m}$ is expected to allow root exudates and $\mathrm{S}$. Typhimurium to pass through it, while the $0.2 \mu \mathrm{m}$ pores would allow the passage of root exudates but not of S. Typhimurium. The polythene sheet was used to completely block the migration of both bacteria and root exudates. The inner space was filled with sterile potting mixture and the outer with treatment with either WT or with flagella mutants ( $\sim 10^{8}$ cells/g soil). A 10-day-old seedling was planted at the center. In another set, root exudates collected from Arabidopsis root (protocol adapted from Turra et al. 2015) was poured into the sterile region. Root exudates were allowed to diffuse for $24 \mathrm{~h}$. From a few pots, the membrane was removed to bring the contaminated and sterile potting mixture into physical contact. CFUs in the inner layer were estimated after 1 week post-inoculation. Pots poured with $0.1 \%$ glucose were used as a positive control. The ratio of CFUs in the inner layer to the outer layer was calculated to examine the effect of root exudates on bacteria migration.

\section{In vitro motility assay}

Swim agar plates were prepared with $0.3 \%$ (w/v) of agar. Overnight cultures grown in LB were spotted on the swim agar plates and incubated at $26^{\circ} \mathrm{C}$ for different time points (Garai et al. 2016). The images of the plates were taken with an Olympus STYLUS VH520 camera.

\section{Confocal microscopy of vermiculite, perlite and peat} moss manure

Potting mixture components treated with GFP-fluorescing Salmonella were observed under a confocal microscope (Zeiss LSM 710 meta). All the components were thoroughly washed with sterile PBS. It was then dipped in congo red solution (10 $\mathrm{mg} / \mathrm{mL}$ ) to stain the exopolysaccharide for $30 \mathrm{~min}$ and washed again with sterile PBS. Vermiculite and perlite were broken down into small pieces and mounted on a cover slip. Organic matter, being bulkier in nature, was carefully sliced using a sterile scalpel, and mounted over the cover slip. Images were analyzed using the ZEN 2009 light edition platform.

\section{Electron microscopy}

For scanning electron microscopy, the samples were fixed in $2.5 \%$ glutaraldehyde for $12 \mathrm{~h}$ and then washed with PBS three times. Samples were dehydrated in a series of ethanol wash from $30 \%$ to $100 \%$. It was finally dried in vacuum. Gold coating was done using the JOEL-JFC-1100E ion sputtering device and observed under scanning electron microscope, and analyzed by field emission-SEM (FEI Sirion, Eindhoven, The Netherlands). Transmission electron microscopy was done as previously described (Garai et al. 2016). Approximately $2 \mu \mathrm{L}$ of cultures were spotted on a copper grid, stained with $1 \%$ uranyl acetate and visualized under transmission electron microscopy.

\section{Inoculum build-up in soil and its transmission to subsequent plants}

Roots of 10-day-old seedlings were either dipped in either 0.3 OD culture of S. Typhimurium suspended in PBS or only PBS (mock control) for $1 \mathrm{~min}$ and transplanted in the potting mixture (12 seedlings per pot having $300 \mathrm{~g}$ soil). Three plants from each pot were sacrificed on the 20th day after transplanting and bacterial burden was assessed in the root, rosette leaves, stem, flowers and fruits. The remaining nine plants were chopped and mixed with the potting mixture and CFU in soil was assessed. Twelve fresh sterile 10-day-old seedlings were transplanted in the same potting mixture and monitored until 20 days. This 
process was repeated for five more generations of plants in the same soil conditions. Each time, plants of the previous generation were chopped and mixed with the potting mixture and CFU was calculated. This experiment was repeated three times with 12 plants each.

\section{Enumeration of pathogen burden in mice organs and} its transmission to subsequent plants

The plants of the fifth-generation growth in the same potting mixture (as discussed in the previous section) were crushed with mortar and pestle in $10 \mathrm{~mL}$ PBS and the suspension was filtered through a $1 \mathrm{~mm}^{2}$ sieve to remove solid particles. The filtrate obtained was further crushed using glass beads in mini beadbeader. Bacterial load in the filtrate was assessed by CFU. Six- to 8-week-old BALB/c mice were infected orally under aseptic conditions either with the filtrate collected from the infected plants or with the mock-treated plants. For organ infiltration studies, livers and spleens were aseptically removed 4 days after infection and homogenized in $1 \mathrm{~mL}$ ice-cold PBS. Serial dilutions of the homogenate were plated on (Salmonella-Shigella agar) SS agar and the CFUs were calculated per gram weight of organ for liver and per organ for the spleen. The experiment was repeated three times with five mice in each group. The fecal pellets of mice fed with infected filtrate or mock filtrate were collected until the third DPI and the bacterial burden was calculated. Fifteen such fecal pellets were mixed with $50 \mathrm{~g}$ of potting mixture and bacterial load was analyzed. Arabidopsis was transplanted into the mixture (five plants per pots) and the bacterial burden was monitored in the root, leaves and fruit as explained above. The experiment was repeated three times with five replicates.

\section{Soil particle size analysis}

Particle size analysis was done by international pipette method as described by Jackson (1973). Air-dry soil (<2 mm) was treated with hydrogen peroxide to remove organic matter. The treated soil was dispersed using a mechanical stirrer with the addition of sodium hexametaphosphate as a dispersing agent. The dispersed solution was passed through a 300 mesh sieve to separate sand particles. The suspension obtained after sieving was analyzed for silt and clay.

\section{Organic carbon measurement}

The dry soil sample was powdered using agate pestle and mortar to pass through a $0.2 \mathrm{~mm}$ sieve. A known weight of the powdered sample was treated with a known volume of standard $\mathrm{K}_{2} \mathrm{Cr}_{2} \mathrm{O}_{7}$ and concentrated $\mathrm{H}_{2} \mathrm{SO}_{4}$. The unused $\mathrm{K}_{2} \mathrm{Cr}_{2} \mathrm{O}_{7}$ was quantified by back titration with standard ferrous ammonium sulphate using ferroin indicator (Walkley and Black 1934).

\section{Ethics statement}

All soil inoculation experiments with tomato and Arabidopsis were conducted as per the standard operating procedures decided by Institutional Biosafety Committee (Ref. IBSC/IISc/DC/07/2017). All animal experiments were reviewed and approved by the Institutional Animal Ethics Committee (IAEC) constituted as per the article number 13 of the CPCSEArules, laid down by Government of India at the IISc, Bangalore, India (Acts, Rules and Amendments no. 59 of 1960). IEAC Registration Number: 48/1999/CPCSEA; Project No.: CAF/Ethics/2014 sanctioned by Chairman: Prof. D N Rao and CPCSEA Nominee: Dr M. R. Gajendragad to Prof. Dipshikha Chakravortty.

\section{Statistical analysis}

To determine whether the bacterial populations in root differed between WT and $\Delta e n v Z / o m p R, \Delta c s g D$ and $\triangle b c s A$, it was logtransformed and one-way ANOVA with Bonferroni post hoc test was performed using Graph Pad Prism to check the significance of CFUs from WT and the mutants. One-way ANOVA followed by Bonferroni post hoc test was performed to check the significance of CFUs of WT and mutants for ex situ motility and soil adhesion. For in vitro soil adhesion, one-way ANOVA with Bonferroni post hoc test was performed to analyze the bacterial retention after $1 \mathrm{~h}$ and $24 \mathrm{~h}$. For comparison of ex situ motility among various strains, multivariate ANOVA was used followed by Tukey's post hoc test. For potting mixture component mixing experiments, all comparisons were made with $1 \times$ mixing using one-way ANOVA. Mann-Whitney test was used to analyze the CFUs obtained from mice liver and spleen.

\section{RESULTS}

\section{Ex situ CFU profiling of Salmonella based on plant proximity}

To test the viability of Salmonella in different regions around a pot-grown plant, we determined the CFU in the non-rhizosphere (edges of the pot), rhizosphere (in the vicinity of roots after uprooting them), rhizoplane (immediate surface of the roots) and root tissue at 20 DPI. Ralstonia solanacearum and E. coli were used as a phytopathogen and non-phytopathogen control, respectively, as described previously (Karmakar et al. 2018). The CFU of S. Typhimurium was significantly lower than that of $R$. solanacearum in non-rhizosphere and rhizosphere, but comparable to that of $E$. coli. On the other hand, the S. Typhimurium CFU in the rhizoplane and tissue was significantly higher than that of E. coli, but was comparable to that of $R$. solanacearum. Thus, we found that, as the proximity of the Salmonella towards the plant increases, its abundance was comparable to that of a phytopathogen (Fig. 1). This is possible due to the nutrient-rich root exudates secreted by the plants. Since the bacteria were homogeneously mixed with the soil, it was difficult to analyze whether the increased CFU near the plant root is because of the enrichment of bacterial population already present in the vicinity or due to the migration of bacteria from the distant region of soil to the rhizoplane.

Horizontal migration of Salmonella towards the plant is mediated by root exudates

In order to examine the migration of bacteria from the nonrhizospheric to the rhizospheric region, we used membranous filters of different pore sizes to radially separate the S. Typhimurium-inoculated potting mixture from the noninoculated one (supplementary Fig. S2, available online). A rise in bacterial population in the inner layer was observed in the case of pots with a $6 \mu \mathrm{m}$ filter (which allows the passage of both root exudates and the bacteria) but not with a $0.2 \mu \mathrm{m}$ filter (which blocks bacteria) or polythene sheet (which blocks both bacteria and root exudates) (supplementary Fig. S3a, available online). Mutant S. Typhimuirum with defects in flagella synthesis, i.e. $\Delta$ fliC and $\Delta$ fliC $\Delta$ fljB, showed lower CFU as compared 


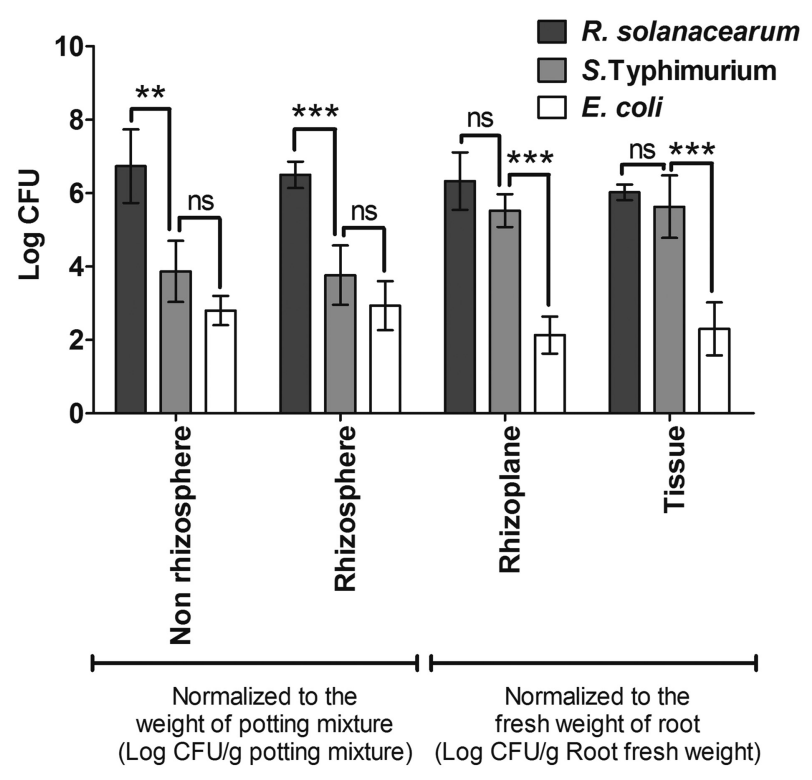

Figure 1. CFU of Salmonella in soil and plant in comparison with R. solanacearum (phytopathogen) and E. coli (non-phytopathogen). One-way ANOVA followed by Bonferroni post hoc test was used to analyze the results. CFU of Salmonella was compared with either $R$. solanacearum or E. coli across the soil (non-rhizosphere and rhizosphere) and plant (rhizoplane and root tissue). ${ }^{* * *} P<0.001$; ${ }^{* *} P<0.01$; ns, $P>0.05$.

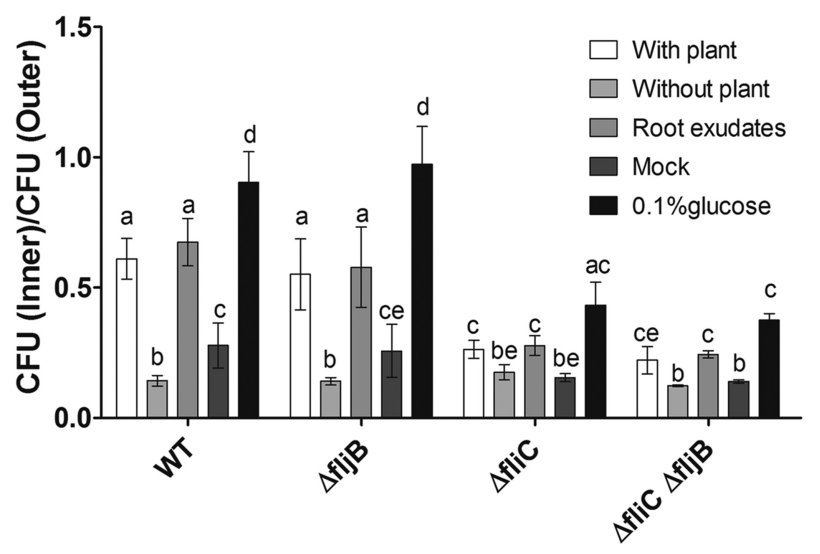

Figure 2. Migration index expressed as CFU in inner layer/CFU in outer layer (1 week post-inoculation) for Salmonella WT and mutants for flagella synthesis in presence and absence of either the plant or treatment with root exudate. Each experiment was repeated three times with three replicates each. Multivariate ANOVA followed by Tukey test was used to analyze the data. Different letters represent significance at $P<0.05$.

with the WT bacteria in the inner layer, but not in $\Delta$ fljB (supplementary Figs S4 and S5, available online). Pots without plants had comparatively fewer bacteria in the inner zone as compared with those with plants (Fig. 2). Enhanced migration of bacteria towards the inner layer was also observed even when root exudates were poured in the centre of the pot, instead of sowing a plant, which was significantly reduced in the case of the $\Delta$ flic and $\Delta$ fliC $\Delta$ fljB mutant (Fig. 2). This suggests that root exudates act as an attractant for the migration of S. Typhimurium, which is aided by the flagella. In the proximity-based experiment, we could not address whether the enrichment of $S$. Typhimurium in the rhizoplane was because of the rapid proliferation of bacteria (due to secreted root exudates) or due to enhanced migration of bacteria towards the rhizoplane. The barrier-based approach revealed the in situ motility of S. Typhimurium in soil. By plotting a growth curve in the Arabidopsis root exudates it was confirmed that none of the strains had proliferation defects (supplementary Fig. S6, available online). Therefore, the lower CFU of mutants in the inner layer is not because of a defect in growth, but because of reduced motility.

\section{Organic matter in soil is essential for the retention of} Salmonella in the soil

In order to check the preferred site of adhesion and colonization amongst the component of potting mixture, we used different microscopy-based approach to visualize the adhesion of Salmonella. A GFP-expressing S. Typhimurium was used to investigate its attachment to the individual components of the potting mixture (vermiculite, perlite and peat moss manure) and was analyzed using confocal microscopy. More GFP-positive cells were observed attached to peat moss manure than vermiculite and perlite (Fig. 3A). This was further validated by electron microscopy. The scanning electron micrograph showed bacterial cells adhered to vermiculite, perlite and manure, but not in the mock-treated controls (Fig. 3B). To test whether these bacterial cells were indeed Salmonella, we serially diluted the samples and plated them on selective growth media. Although a timedependent increase in Salmonella CFU was observed in all the soil components (Fig. 3C), the higher bacterial count was found in the organic manure compared with vermiculite and perlite. This implies that the organic manure is the preferred site of adhesion. We further verified the adherence of bacteria to tomato field soil, which has a sandy clay loam texture (supplementary Fig. S7a, available online), after treating it with varying amounts of perlite, vermiculite and peat moss manure. Addition of peat moss manure to the field soil increased the organic carbon content (supplementary Fig. S7b, available online). An increasing proportion of organic matter (peat moss manure) in the soil mixture showed a positive correlation with bacterial retention (Fig. 3D). Thus, the amendment of organic manure to the soil increases the attachment of Salmonella as compared with the inorganic components (perlite and vermiculite).

Role of the two-component system (TCS) and exopolysaccharides (EPS) in the adhesion of Salmonella to the soil components

Bacterial attachment to surfaces requires the synthesis of exopolysaccharides (EPS) triggered by osmotic stress through the two-component system (TCS) (Stanley and Lazazzera 2004). Environmental signals are perceived by the membrane-bound sensor kinase envZ, which phosphorylates the response regulator ompR that controls the downstream processes such as EPS synthesis and biofilm formation (Gerstel, Kolb and Romling 2006) (supplementary Fig. S8a, available online). We used TCS ( $\Delta e n v Z / o m p R)$ and EPS ( $\triangle b c s A$ and $\Delta c s g D)$ mutants to evaluate the role of this signaling pathway in bacterial attachment and retention in potting mixture. We observed that the $S$. Typhimurium mutants lacking genes for envZ/ompR (encoding the TCS), $\operatorname{csg} D$ (transcription factor that regulates biofilm formation) and bcsA (cellulose synthase), which are incapable of synthesizing EPS, show less pellicle formation on biofilm media than the WT bacteria (supplementary Fig. S8b and c, available 

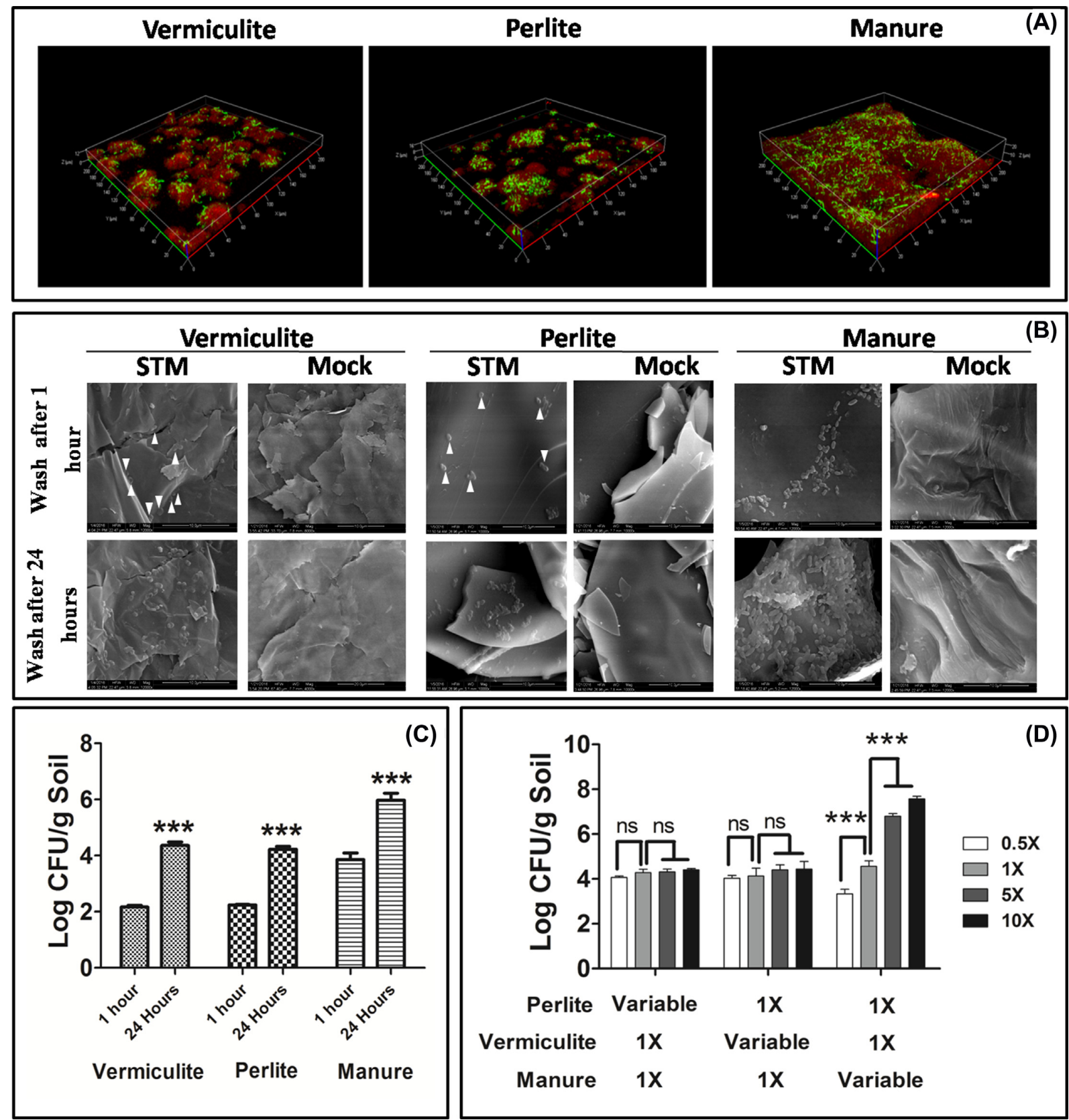

Figure 3. Adhesion of Salmonella to the soil particles. (A) Representative confocal image of vermiculite, perlite and manure with Salmonella expressing GFP (green) and EPS is stained by congo red (red). (B) Representative images showing adhered Salmonella cells on vermiculite, perlite and organic manure (peat moss) post-washing after $1 \mathrm{~h}$ and $24 \mathrm{~h}$. (C) Enumeration of CFU on each particle by dilution plating. One-way ANOVA was used to analyze the data. Comparison was done between CFU obtained after $1 \mathrm{~h}$ and $24 \mathrm{~h} .{ }^{* * *} \mathrm{P}<0.001$. (D) CFU analysis upon changing individual components (inorganic: vermiculite and perlite; organic: peat moss manure) of the soil. Each experiment was repeated three times with three replicates each. One-way ANOVA followed by Bonferroni post hoc test was used to analyze the data. Comparison was done with $1 \times$ mixture of component of potting mixture with other mixing ratios. ${ }^{* * *} P<0.001 ; n s, P>0.05$.

online). To test whether the adhesive properties of EPS (Flemming and Wingender 2010) help the bacterial cells bind to the soil components, we pretreated the potting mixture with WT or mutant bacteria and washed them thoroughly after $24 \mathrm{~h}$. Post-washing, the potting mixture was visualized under an electron microscope. Comparatively fewer cells of mutant bacteria were found adhered to the potting mixture as compared with the WT cells (Fig. 4A). These mutants did not colonize the Arabidopsis root as efficiently as the WT bacteria (supplementary Fig. S9, available online). However, neither the mutant nor the
WT showed any growth defect in the root exudates collected from the Arabidopsis, thus indicating that the reduced colonization is not because of compromised survival (supplementary Fig. S10, available online). This was further validated by the ex situ adhesion assay. The mutants lacking the ability to adhere to the components of potting mixture ( $\Delta e n v Z / o m p R, \Delta \operatorname{csg} D$ and $\Delta b c s A$ ) were washed away from the top layer and drained out via flowthrough, as opposed to the WT, which retained even after washing (Fig. 4B and C), suggesting the importance of these genes in adhesion to soil. 

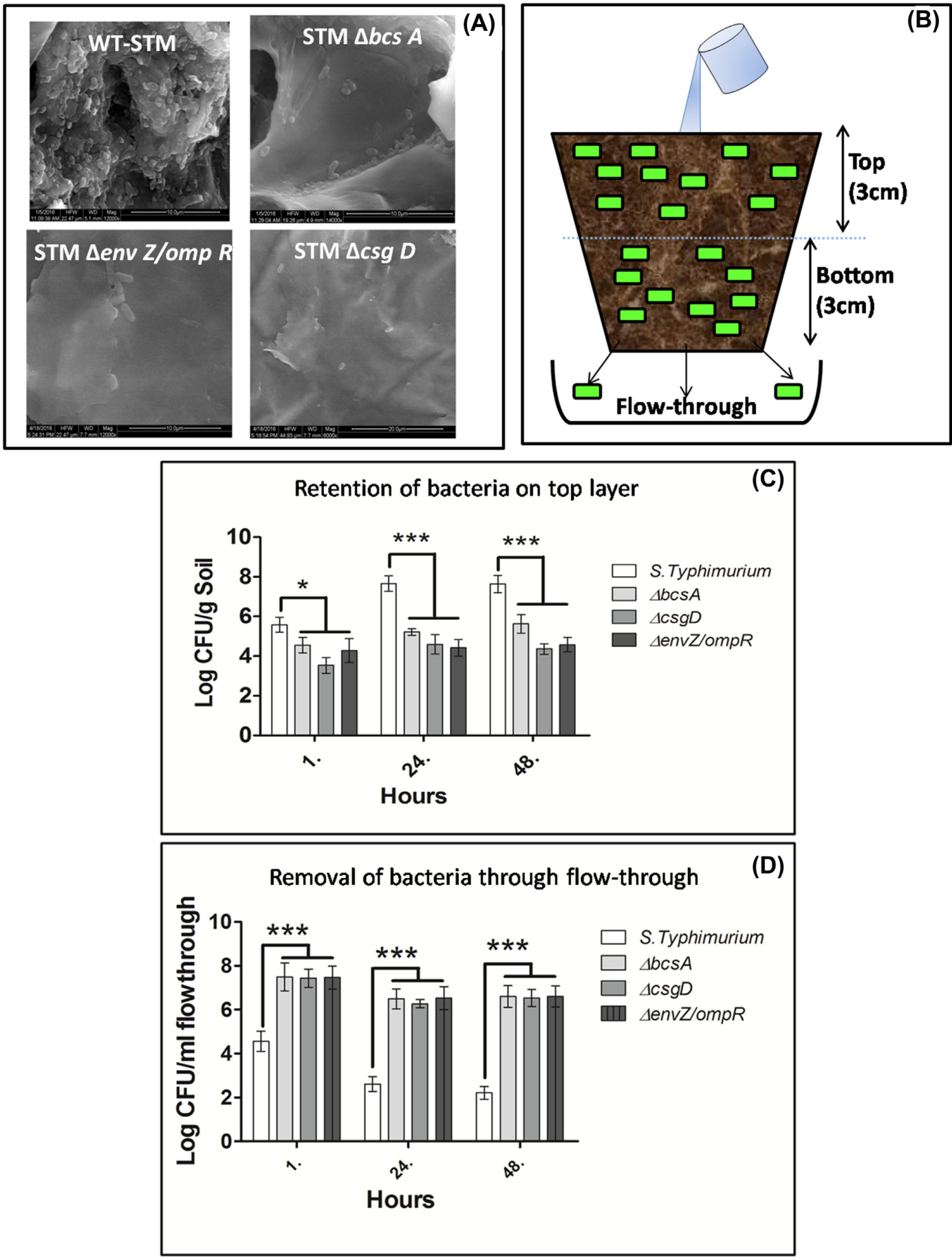

Figure 4. Role of TCS and EPS genes in soil adhesion. (A) Representative images showing adhered Salmonella cells on soil particles $24 \mathrm{~h}$ post-inoculation. (B) Schematic representing the experimental setup for bacterial soil retention and flow-throgh. (C) The CFU retained in top layer and (D) removed via flow-though for Salmonella WT, TCS and EPS mutants at different times. One-way ANOVA followed by Bonferroni post hoc test was used to analyze the results. CFU values between WT bacteria are compared with TCS and EPS mutant bacteria. ${ }^{* * *} P<0.001 ;{ }^{*} P<0.05$.

When the CFU of these mutants were compared with WT, a significant decrease in the number of bacteria retained after the wash was observed as the proximity towards the plant increased (Fig. 5). In all four regions, the colonization by the WT Salmonella was significantly higher than the TCS and EPS mutants. This result shows the importance of environment sensing by TCS genes and the biofilm-forming EPS genes in soil persistence and plant colonization and invasion.
Inoculum build-up and re-establishment of infection in plants via passage through murine intermediate

To mimic the build-up of the soil inoculum of Salmonella, we grew six generations of Arabidopsis plants in potting mixture containing the macerated remains of the infected plants from the previous generation (see Materials and methods). We observed a progressive increase in Salmonella CFU after every generation 


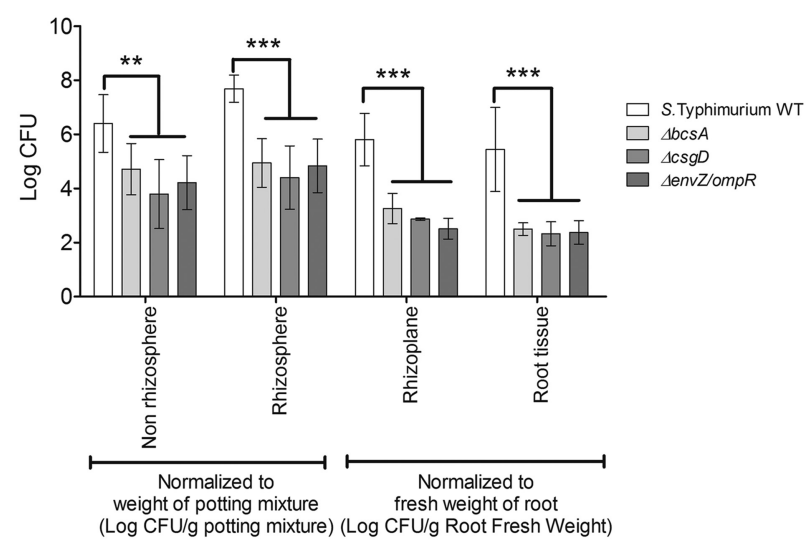

Figure 5. CFU of Salmonella in soil and plant in comparison with biofilm-deficient mutant. One-way ANOVA followed by Bonferroni post hoc test was used to analyze the results. CFU values between WT bacteria are compared with TCS and EPS mutant bacteria across the soil (non-rhizosphere and rhizosphere) and plant (rhizoplane and root tissue). ${ }^{* * *} P<0.001 ;{ }^{* *} P<0.01$.

(Fig. 6A), suggesting the role of fresh organic matter in harboring the Salmonella population in the soil. Sowing of sterile plants in such potting mixture led to an increase in CFU in multiple tissue types (root, leaves, flower and fruits) after every generation (Fig. 6A).

The filtrate collected from the plants grown in this artificial soil, carrying the contaminated plant residues from the previous five generations, was fed to the BALB/c mice, which showed higher Salmonella burden in their liver and spleen (primary organs for Salmonella colonization) and in fecal pellet as compared with those fed with mock filtrate (Fig. 6C-E). Fecal matters collected from both sets of mice were mixed with sterile potting mixture (15 fecal pellet/50 g of pot mix) and the bacterial burden was enumerated (Fig. 6F). Plants grown in artificial soil mixed with this fecal matter from infected mice showed the presence of Salmonella in roots, leaves and fruits (Fig. 6G), thus showing the re-establishment of infection in plants and completing the trans-kingdom cycle.

\section{DISCUSSION}

One of the sources of Salmonella infection is by the consumption of contaminated raw food products such as salad vegetables and fruits (Park et al. 2012; Cui, Walcott and Chen 2017; Koukkidis et al. 2017). Contamination can occur during crop growth, i.e. before harvest, through soil. Survival of Salmonella in the soil is reported to be up to 300 days (Baloda, Christensen and Trajcevska 2001). Greene et al. reported that Salmonella enters the tomato field through contaminated irrigation water (Greene et al. 2008). Root hairs, which are the primary site of nutrient uptake (Gilroy and Jones 2000; Leitner et al. 2010) and also the site of the release of plant exudates (Yan et al. 2004; Holz et al. 2018), are reported to be colonized by Salmonella possibly due to nutrient enrichment in these regions. Schikora et al. also demonstrated that Arabidopsis plants infiltrated with Salmonella develop symptoms such as chlorosis of the leaves, which could be the result of activation of defense-related genes (Schikora et al. 2008).

Migration of bacteria from a distant place towards the root surface is influenced by root exudates. Beneficial organisms like Rhizobia exhibit such chemo-attraction and colonize the root hair (Poole, Ramachandran and Terpolilli 2018). In addition, amino acids and sugars present in the root exudates serve as a nutrient source (Han and Micallef 2016). The Salmonella mutant deficient in the synthesis of flagella grew in the root exudates (comparable to WT). By using membranes of different pore sizes we eliminated the possibility of passive diffusion of bacteria across the artificial soil mixture. The migration of Salmonella was significantly higher when root exudate was allowed to diffuse through the membrane. Our results were in accordance with other reports such as in vitro plate migration on water agar (Barak et al. 2009), capillary-based migration (Klerks et al. 2007) and epiphytic migration via roots (Cooley, Miller and Mandrell 2003). Moreover, the barrier-based approach helped us in the observation of directed migration and nullifies the effect of free-floating bacteria in the soil.

Osmotic stress in the soil is caused by high evapotranspiration (drought) or accumulation of agro-chemicals and salts (salinity) (Karmakar et al. 2015). Both these stresses reduce the water potential of the soil. In the free-living state, many bacteria produce EPS that help colonize the plants (Vlamakis et al. 2013; Karmakar et al. 2015) or evade host defenses (Abramovitch, Anderson and Martin 2006). Salmonella is known to produce cellulose as EPS matrix and biofilms on solid, liquid-solid and liquid-air interfaces (Srinandan et al. 2015). The protective and adhesive nature of the biofilm produced by enteric pathogens is important for their survival (Steenackers et al. 2012). We observed that the biofilm produced by the WT Salmonella is seen on the soil surface within $24 \mathrm{~h}$ post-treatment. Its adhesive property gives an advantage of firm attachment to the soil particles without being washed away by irrigation water. It was also found that the organic component of the soil provides a strong substratum for the bacterial attachment as compared with the inorganic matter. The osmotic stress created by the lowering of the water potential is sensed by the EnvZ/OmpR TCS (Foo et al. 2015). The phosphorylated OmpR binds to the promoter of $\operatorname{csg} \mathrm{D}$ and activates it, resulting in a further induction of cellulose synthesis by bcsA (cellulose synthase), thus forming an extracellular matrix of the biofilm (Gerstel, Kolb and Romling 2006; Liu et al. 2014). Ex situ soil-binding assay and the rhizoplane colonization experiments demonstrated the importance of extracellular matrix in adhesion of Salmonella to the soil. Our results are in accordance with the earlier reports on the attachment to glass (Barak et al. 2007) and quartz (Haznedaroglu et al. 2009). To assess the stability of the biofilm adhesion to the substratum, we analyzed the CFU in the flow-through and the retention in the top layer of the irrigated pot. This experiment was possible only in the Arabidopsis soil mix (perlite:vermiculite:peat moss manure) because of the high porosity. As the field soil became more compacted upon watering, the flow-through could not be obtained. However, the CFU analysis performed for field soil upon treatment with varying amount of inorganic and organic matter confirmed that the organic matter in the field provides a better attachment to Salmonella.

Proximity-based CFU analysis demonstrated the importance of plants in mediating chemo-attraction and enrichment of Salmonella in the rhizosphere (Fig. 1). We had previously shown that root colonists like $R$. solanacearum, when inoculated at the dose of $10^{8} \mathrm{CFU} / \mathrm{g}$ soil, cause immediate death of the plant (Karmakar et al. 2018). We reduced the dose of $R$. solanacearum to $10^{4} \mathrm{CFU} / \mathrm{g}$ potting mixture and found that there is comparatively less death of plants (supplementary Fig. S11, available online). Hence, R. solanacearum was used as the phytopathogen control. Escherichia coli DH5 $\alpha$ did not induce lateral root proliferation and was a poor colonist; hence it was used as the nonphytopathogen control (Karmakar et al. 2018). Parameters like 


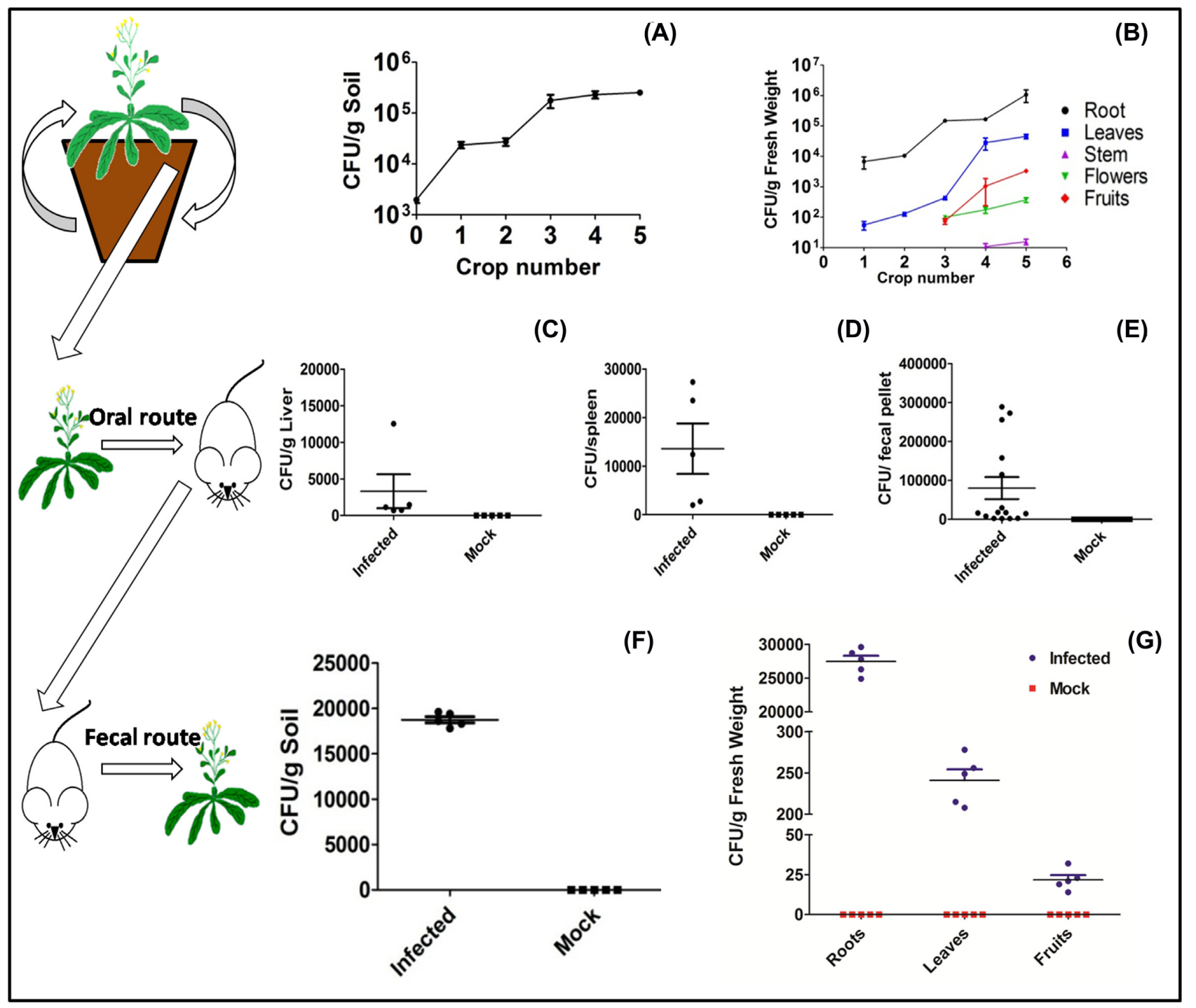

Figure 6. Inoculum build-up in soil and re-establishment of infection in plant via feces of murine intermediate. (A) CFU of Salmonella in soil every generation after mixing the previous crop residue. (B) Organ burden of Salmonella in roots, leaves, flower, fruit and stem. (C and D) CFU of Salmonella in liver and spleen of mice fed with filtrate obtained from infected and mock-treated plants raised for five generations in the same soil. (E) CFU of Salmonella in fecal pellets of mice fed with infected and mock filtrate. (F) CFU of Salmonella in soil mixed with fecal pellet of mice fed with infected filtrate or mock filtrate. (G) Organ burden of plant raised in soil mixed with the fecal matter of mice fed with either infected filtrate or mock filtrate.

biofilm formation and binding to the soil particles are equally important for bacteria to survive in the soil. The mutants lacking TCS and EPS were deficient in the adhesion to soil particles, and their CFUs were significantly fewer than the wild type, demonstrating the importance of these genes in soil survival and plant colonization.

Growing crops in the contaminated fields can lead to infection of the subsequent produce. It had been reported earlier that growing plants in soil that harbors aSalmonella burden because of mixing of previous crop residue results in colonization of the rhizoplane and phyllosphere with the pathogen in the next generation of plant (Barak and Liang 2008). We performed a similar experiment for multiple generations of crops and concluded that mixing of the fresh organic matter into the soil in the form of crop residue enhances the bacterial burden in the soil and its dissemination to the aerial organs with every passing crop generation. We also demonstrated that after a successful colonization of plant roots and aerial organs, Salmonella shows transmission to the animal hosts. Schikora et al. reported the infection of human cell lines and mice with Salmonella of plant origin (Schikora et al. 2011). We used the filtrate from the infected plants as a source of inoculum and fed it to BALB/c mice, which accumulated Salmonella burden in liver, spleen and fecal matter. The fecal pellets were used to re-establish the infection in new plants, thus completing the trans-kingdom cycle.

We used a systematic approach to study the transmission of Salmonella through members of two different kingdoms, i.e. the kingdom Plantae (Arabidopsis) and the kingdom Animalia (mouse). Our results demonstrate selective migration of Salmonella from the soil towards the root tissue. Salmonella is 
known to survive as an endophyte and epiphyte on Arabidopsis root (Cooley, Miller and Mandrell 2003). It is also known to suppress the plant defense responses and oxidative burst (Shirron and Yaron 2011), and hence can remain undetected. It can reach the edible plant parts which, upon consumption, can cause disease in animals. This could be associated with various health risks and should be taken into account, as reports indicate that edible crops like tomato (Guo et al. 2001, 2002; Gu et al. 2013; $\mathrm{Gu}$, Cevallos-Cevallos and van Bruggen 2013), lettuce (Klerks et al. 2007) and basil (Berger et al. 2009) can be associated with Salmonella. Our report fills the gap in the knowledge of the processes occurring between the soil attachment of bacteria and the transmission to plants. Based on this, strategies can be developed to reduce the Salmonella burden in soil, possibly by using competing beneficial micro-organisms.

\section{SUPPLEMENTARY DATA}

Supplementary data are available at FEMSEC Journal online.

\section{ACKNOWLEDGEMENTS}

Strain STM $\triangle e n v Z / o m p R$ was provided by Dr Michel Hensel (Division Microbiology, University Osnabrück, Germany). The Departmental Confocal facility (Microbiology and Cell Biology) and Divisional electron microscopy facilty (Indian Institute of Science) are acknowledged. Prof. Tanweer Hussain and Prof. Somnath Dutta, IISc, are acknowledged for their help in acquiring the images using transmission electron microscopy. Ms Anusha and Ms Satyawati are acknowledged for their help in image acquisition. K.K. and A.V.N. acknowledge IISc Fellowship from MHRD, Govt of India. The authors would like to acknowledge the research grants Life Science Research Board (LSRB0008), and DBT-IISc partnership program for advanced research in biological sciences and bioengineering to D.C. Infrastructure support from ICMR (Center for Advanced Study in Molecular Medicine), DST (FIST) and UGC (special assistance) is acknowledged. D.C. received a DAE SRC outstanding Investigator award and funds.

Conflicts of interest. None declared.

\section{REFERENCES}

Abramovitch RB, Anderson JC, Martin GB. Bacterial elicitation and evasion of plant innate immunity. Nat Rev Mol Cell Biol 2006;7:601-11.

Baloda SB, Christensen L, Trajcevska S. Persistence of a Salmonella enterica serovar typhimurium DT12 clone in a piggery and in agricultural soil amended with Salmonellacontaminated slurry. Appl Environ Microbiol 2001;67:2859-62.

Barak JD, Gorski L, Liang AS et al. Previously uncharacterized Salmonella enterica genes required for swarming play a role in seedling colonization. Microbiology 2009;155:3701-9.

Barak JD, Jahn CE, Gibson DL et al. The role of cellulose and Oantigen capsule in the colonization of plants by Salmonella enterica. Mol Plant Microbe Interact 2007;20:1083-91.

Barak JD, Kramer LC, Hao LY. Colonization of tomato plants by Salmonella enterica is cultivar dependent, and type 1 trichomes are preferred colonization sites. Appl Environ Microbiol 2011;77:498-504.

Barak JD, Liang A, Narm KE. Differential attachment to and subsequent contamination of agricultural crops by Salmonella enterica. Appl Environ Microbiol 2008;74:5568-70.
Barak JD, Liang AS. Role of soil, crop debris, and a plant pathogen in Salmonella enterica contamination of tomato plants. PLoS One 2008;3:e1657.

Berger CN, Shaw RK, Brown DJ et al. Interaction of Salmonella enterica with basil and other salad leaves. ISME J 2009;3:2615.

Bhardwaj V, Meier S, Petersen LN et al. Defence responses of Arabidopsis thaliana to infection by Pseudomonas syringae are regulated by the circadian clock. PLoS One 2011;6:e26968.

Bodenhausen N, Horton MW, Bergelson J. Bacterial communities associated with the leaves and the roots of Arabidopsis thaliana. PLoS One 2013;8:e56329.

Brandl MT. Fitness of human enteric pathogens on plants and implications for food safety. Annu Rev Phytopathol 2006;44:367-92.

Cabello JV, Giacomelli JI, Gomez MC et al. The sunflower transcription factor HaHB11 confers tolerance to water deficit and salinity to transgenic Arabidopsis and alfalfa plants. $J$ Biotechnol 2017;257:35-46.

Cooley MB, Miller WG, Mandrell RE. Colonization of Arabidopsis thaliana with Salmonella enterica and enterohemorrhagic Escherichia coli 0157:H7 and competition by Enterobacter asburiae. Appl Environ Microbiol 2003;69:4915-26.

Cowles KN, Willis DK, Engel TN et al. Diguanylate cyclases AdrA and STM1987 regulate salmonella enterica exopolysaccharide production during plant colonization in an environment-dependent manner. Appl Environ Microbiol 2015;82:1237-48.

Cox CE, Brandl MT, de Moraes MH et al. Production of the plant hormone auxin by Salmonella and its role in the interactions with plants and animals. Front Microbiol 2018;8:2668.

Cui Y, Walcott R, Chen J. Differential attachment of salmonella enterica and enterohemorrhagic escherichia coli to alfalfa, fenugreek, lettuce, and tomato seeds. Appl Environ Microbiol 2017;83:1-12.

Datsenko KA, Wanner BL. One-step inactivation of chromosomal genes in Escherichia coli K-12 using PCR products. Proc Natl Acad Sci USA 2000;97:6640-5.

Flemming HC, Wingender J. The biofilm matrix. Nat Reu Microbiol 2010;8:623-33.

Foo YH, Spahn C, Zhang $\mathrm{H}$ et al. Single cell super-resolution imaging of E. coli OmpR during environmental stress. Integr Biol (Camb) 2015;7:1297-308.

Garai P, Lahiri A, Ghosh D et al. Peptide utilizing carbon starvation gene yjiY is required for flagella mediated infection caused by Salmonella. Microbiology 2015:100-16.

Garai P, Lahiri A, Ghosh D et al. Peptide utilizing carbon starvation gene yjiY is required for flagella mediated infection caused by Salmonella. Microbiology 2016;162:100-16.

Gerstel U, Kolb A, Romling U. Regulatory components at the csgD promoter-additional roles for OmpR and integration host factor and role of the $5^{\prime}$ untranslated region. FEMS Microbiol Lett 2006;261:109-17.

Gilroy S, Jones DL. Through form to function: root hair development and nutrient uptake. Trends Plant Sci 2000;5: 56-60.

Greene SK, Daly ER, Talbot EA et al. Recurrent multistate outbreak of Salmonella Newport associated with tomatoes from contaminated fields, 2005. Epidemiol Infect 2008;136:157-65.

Gu G, Cevallos-Cevallos JM, Vallad GE et al. Organically managed soils reduce internal colonization of tomato plants by Salmonella enterica serovar Typhimurium. Phytopathology 2013;103:381-8. 
$\mathrm{Gu}$ G, Cevallos-Cevallos JM, van Bruggen AH. Ingress of Salmonella enterica Typhimurium into tomato leaves through hydathodes. PLoS One 2013;8:e53470.

Gu G, Hu J, Cevallos-Cevallos JM et al. Internal colonization of Salmonella enterica serovar Typhimurium in tomato plants. PLoS One 2011;6:e27340.

Guo X, Chen J, Brackett RE et al. Survival of salmonellae on and in tomato plants from the time of inoculation at flowering and early stages of fruit development through fruit ripening. Appl Environ Microbiol 2001;67:4760-4.

Guo X, van Iersel MW, Chen $J$ et al. Evidence of association of salmonellae with tomato plants grown hydroponically in inoculated nutrient solution. Appl Environ Microbiol 2002;68:3639-43.

Han S, Micallef SA. Environmental metabolomics of the tomato plant surface provides insights on Salmonella enterica colonization. Appl Environ Microbiol 2016;82:3131-42.

Hao LY, Willis DK, Andrews-Polymenis $\mathrm{H}$ et al. Requirement of siderophore biosynthesis for plant colonization by Salmonella enterica. Appl Environ Microbiol 2012;78:4561-70.

Haznedaroglu B, Kim H, Bradford S et al. Relative transport behavior of Escherichia coli 0157: H7 and Salmonella enterica serovar pullorum in packed bed column systems: Influence of solution chemistry and cell concentration. Environ Sci Technol 2009;43:1838-44.

Hernandez-Reyes C, Adam S. Salmonella, a cross-kingdom pathogen infecting humans and plants. FEMS Microbiol Lett 2013;343:1-7.

Holden NJ, Jackson RW, Schikora A. Editorial on plants as alternative hosts for human and animal pathogens. Front Microbiol 2015;6:397.

Holz M, Zarebanadkouki M, Kuzyakov Y et al. Root hairs increase rhizosphere extension and carbon input to soil. Ann Bot 2018;121:61-9.

Hua L, Challa GS, Subramanian S et al. Genome-wide identification of drought response genes in soybean seedlings and development of biomarkers for early diagnoses. Plant Mol Biol Rep 2018;36:350-62.

Hu Y, Gao YR, Wei W et al. Strawberry MOTHER OF FT AND TFL1 regulates seed germination and post-germination growth through integrating GA and ABA signaling in Arabidopsis. Plant Cell Tiss Org 2016;126:343-52.

Jackson ML. Soil Chemical Analysis. London: Constable, 1973.

Jantsch J, Chikkaballi D, Hensel M. Cellular aspects of immunity to intracellular Salmonella enterica. Immunol Rev 2011;240:185-95.

Karmakar K, Nath U, Nataraja KN et al. Root mediated uptake of Salmonella is different from phyto-pathogen and associated with the colonization of edible organs. BMC Plant Biol 2018;18:344.

Karmakar K, Rana A, Rajwar A et al. Legume-Rhizobia Symbiosis Under Stress. India: Springer India, 2015.

Klerks MM, Franz E, van Gent-Pelzer M et al. Differential interaction of Salmonella enterica serovars with lettuce cultivars and plant-microbe factors influencing the colonization efficiency. ISME J 2007;1:620-31.

Koukkidis G, Haigh R, Allcock N et al. Salad leaf juices enhance Salmonella growth, colonization of fresh produce, and virulence. Appl Environ Microbiol 2017;83:e02416-16.

Leitner D, Klepsch S, Ptashnyk M et al. A dynamic model of nutrient uptake by root hairs. New Phytol 2010;185:792-802.

Liu Z, Niu H, Wu S et al. CsgD regulatory network in a bacterial trait-altering biofilm formation. Emerg Microbes Infect 2014;3:e1.
Lv P, Liu C, Rao Z. Review on clay mineral-based form-stable phase change materials: preparation, characterization and applications. Renew Sust Energ Rev 2017;68:707-26.

Mohle-Boetani JC, Farrar J, Bradley P et al. Salmonella infections associated with mung bean sprouts: epidemiological and environmental investigations. Epidemiol Infect 2009;137:35766.

Nsoesie EO, Kluberg SA, Brownstein JS. Online reports of foodborne illness capture foods implicated in official foodborne outbreak reports. Preu Med 2014;67:264-9.

Pansu M, Gautheyrou J. Handbook of Soil Analysis: Mineralogical, Organic and Inorganic Methods. Berlin: Springer Science \& Business Media, 2007.

Park S, Szonyi B, Gautam R et al. Risk factors for microbial contamination in fruits and vegetables at the preharvest level: A systematic review. J Food Prot 2012;75:2055-81.

Poole P, Ramachandran V, Terpolilli J. Rhizobia: from saprophytes to endosymbionts. Nat Rev Microbiol 2018;16: 291-303.

Schikora A, Carreri A, Charpentier E et al. The dark side of the salad: Salmonella typhimurium overcomes the innate immune response of Arabidopsis thaliana and shows an endopathogenic lifestyle. PLoS One 2008;3:e2279.

Schikora A, Garcia AV, Hirt H. Plants as alternative hosts for Salmonella. Trends Plant Sci 2012;17:245-9.

Schikora A, Virlogeux-Payant I, Bueso E et al. Conservation of salmonella infection mechanisms in plants and animals. PLoS One 2011;6:e24112.

Shirron N, Yaron S. Active suppression of early immune response in tobacco by the human pathogen Salmonella typhimurium. PLoS One 2011;6:e18855.

Sivapalasingam S, Barrett E, Kimura A et al. A multistate outbreak of Salmonella enterica Serotype Newport infection linked to mango consumption: impact of water-dip disinfestation technology. Clin Infect Dis 2003;37:1585-90.

Smalla K, Wieland G, Buchner A et al. Bulk and rhizosphere soil bacterial communities studied by denaturing gradient gel electrophoresis: plant-dependent enrichment and seasonal shifts revealed. Appl Environ Microbiol 2001;67:4742-51.

Srinandan CS, Elango M, Gnanadhas DP et al. Infiltration of matrix-non-producers weakens the salmonella biofilm and impairs its antimicrobial tolerance and pathogenicity. Front Microbiol 2015;6:1468.

Stanley NR, Lazazzera BA. Environmental signals and regulatory pathways that influence biofilm formation. Mol Microbiol 2004;52:917-24.

Steenackers H, Hermans K, Vanderleyden J et al. Salmonella biofilms: An overview on occurrence, structure, regulation and eradication. Food Res Int 2012;45:502-31.

Turra D, El Ghalid M, Rossi F et al. Fungal pathogen uses sex pheromone receptor for chemotropic sensing of host plant signals. Nature 2015;527:521-4.

Van Beneden CA, Keene WE, Strang RA et al. Multinational outbreak of Salmonella enterica serotype Newport infections due to contaminated alfalfa sprouts. JAMA 1999;281:158-62.

Vlamakis H, Chai Y, Beauregard P et al. Sticking together: building a biofilm the Bacillus subtilis way. Nat Rev Microbiol 2013;11:157-68.

Walkley A, Black IA. An examination of the Degtjareff method for determining soil organic matter, and a proposed modification of the chromic acid titration method. Soil Sci 1934;37:2938.

Ward LR, Maguire C, Hampton MD et al. Collaborative investigation of an outbreak of Salmonella enterica serotype Newport 
in England and Wales in 2001 associated with ready-to-eat salad vegetables. Commun Dis Public Health 2002;5:301-4.

Wiedemann A, Virlogeux-Payant I, Chaussé A-M et al. Interactions of Salmonella with animals and plants. Front Microbiol 2015;5:791.
Yan XL, Liao H, Beebe SE et al. QTL mapping of root hair and acid exudation traits and their relationship to phosphorus uptake in common bean. Plant Soil 2004;265:17-29. 